\title{
An Approach to Characterizing the Type of Combined Environmental Toxicity Based on Epidemiologically Assessed Exposure-Response Relationships
}

\author{
Boris A. Katsnelson ${ }^{*, 1}$, Larisa I. Privalova ${ }^{1,2}$, Anatoly N. Varaksin ${ }^{3}$, Julia I. Kazmer ${ }^{1}$, Ekaterina P. \\ Kireyeva $^{1}$ and Vladimir G. Panov ${ }^{3}$ \\ ${ }^{I}$ Medical Research Center for Prophylaxis and Health Protection in Industrial Workers, Ekaterinburg, Russia \\ ${ }^{2}$ Urals Regional Center for Environmental Epidemiology, Ekaterinburg, Russia \\ ${ }^{3}$ Institute of Industrial Ecology, the Urals Branch of the Russian Academy of Sciences, Ekaterinburg, Russia
}

\begin{abstract}
Using a dataset obtained in an earlier published epidemiological study that revealed the dependence of the probability of subclinical kidney damage in 260 children on the concentration of lead and cadmium in their urine, we have tested some methodological approaches to assessing the type of combined nephrotoxicity produced by these two metals. We have found that the environmentally caused damage to kidneys in children from lead and cadmium is less than additive (manifestation of toxicological antagonism).

Given the subadditivity (antagonism) of the damaging effect of lead and cadmium on kidneys as demonstrated on the basis of epidemiological data, we believe that the summation of corresponding risks is a sufficiently conservative principle creating an additional margin of safety and limiting the uncertainty of risk assessment on the whole.

Of theoretical interest is the demonstrated consistency of this assessment of the type of combined toxicity irrespective of whether it is carried out on the basis of the paradigm of effect additivity or dose additivity. This enables us to speak in favor of considering the so-called Bliss independence and Loewe additivity as complementary simplified models of the same fairly complex process rather than essentially different biological phenomena. The example studied suggests that approaches to the analysis of epidemiological data for the purpose of assessing combined risk should be tested using each of these models rather than restricting it to one of them based on an a priori choice.
\end{abstract}

Keywords: Case-control study, nephrotoxicity, cadmium, lead.

\section{INTRODUCTION}

In health risk assessment one, as a rule, uses exposureresponse relationships established (either in animal experiments or, much better, in epidemiological studies) for a single quasi-isolated toxicant, while in reality the environment is contaminated with a multitude of hazardous substances, some of them having similar adverse effects.

The combined effect of toxic substances is an issue that is interesting for both toxicology and environmental epidemiology; the latter, however, usually considers it only as part of the analysis of the dependence of this or that adverse health outcome on a multitude of various risk factors within the paradigm of a web of causation. Although, in practice, neither of these two areas of knowledge deals with problems which are more complex than the dependence of an effect on (or a response to) a combination of just two harmful factors, the approaches employed in them for this

*Address correspondence to this author at the Toxicology \& Bioprophylaxis, Medical Research Center for Prophylaxis and Health Protection in Industrial Workers; 30 Popov Str, Ekaterinburg 620014 Russia; Tel: 007-343-3717721; Fax: 007-343-3717-740; E-mails: bkaznelson@etel.ru,

bkaznelson@ymrc.ru, honored_sci@yahoo.com purpose are not quite compatible, to the extent that even the conceptual frameworks prevalent in each of these areas are dissimilar.

Indeed, rather than mentioning combined toxicity, an epidemiologist would more often than not talk about "interaction" and "effect modification", sometimes drawing a clear distinction between these concepts and considering complex variants of their combinations, the extreme ones being effect modification with no interaction, and interaction with no modification [1].

Toxicology will preferably operate the concepts of "additivity", "synergism" (or synergy, or potentiation, or superadditivity), and "antagonism" (or subadditivity), which are sometimes used in epidemiological dictionaries and glossaries as well. However, the contents of these basic concepts and types of combined toxicity corresponding to them are highly varied. A comprehensive review of the literature is not among our objectives, and we shall limit ourselves to a brief and somewhat simplified review of two fundamental concepts which are often used in publications on this theme, i.e. Bliss independence and Loewe additivity $[2,3]$.

Bliss independence assumes the dependence of the same effect on the action of two or more substances at different 
biological sites so that the effect of a chemical at a particular dose is independent of the presence of another chemical, but the combined effect can be modified by interaction between the effects. Thus, the additivity of effects produced by a combination of two agents $\mathrm{A}$ and $\mathrm{B}$ corresponds to the equation $E_{A B}=E_{A}+E_{B}-E_{A} E_{B}$. If the actually observed effect of this combination is higher or lower than the thus specified expected effect $E_{A B}$ for zero interaction, we deal with synergy or antagonism, respectively. Strictly speaking, this equation which is but a transformation of the standard expression for summing two independent probabilities: $\mathrm{p}_{\mathrm{AB}}=$ $\mathrm{p}_{\mathrm{A}}+\left(1-\mathrm{p}_{\mathrm{A}}\right) \mathrm{p}_{\mathrm{B}}$ is correct only when $\mathrm{E}$ is a proportion of a population or a group which sustained the damage under consideration (disease, death, etc.), i.e. what epidemiology defines as "response". It would not have any mathematical sense if the effect is understood as a quantitative shift in this or that index of the organism's status caused by factors A and $\mathrm{B}$ due to independent mechanisms. In this case evidently $\mathrm{E}_{\mathrm{AB}}$ should be equal simply to $\left(\mathrm{E}_{\mathrm{A}}+\mathrm{E}_{\mathrm{B}}\right)$. Note that when $\left(\mathrm{p}_{\mathrm{A}}\right.$ $\left.+\mathrm{p}_{\mathrm{B}}\right) \leq 1$ this simplified equation $\left(\mathrm{E}_{\mathrm{AB}}=\mathrm{E}_{\mathrm{A}}+\mathrm{E}_{\mathrm{B}}\right)$ is sometimes used even in respect to "responses".

On the contrary, Loewe additivity proceeds from the assumption that two or more chemicals act on the same biological site by the same mechanisms of action, and differ only in their potency. In other words, A and B are, in fact, considered as acting as the same substance and, consequently, as not entering into interaction. Thus, when $\mathrm{D}_{\mathrm{A}}$ and $D_{B}$ are effective doses of these chemicals (e.g. their $\mathrm{LD}_{50}$ ) then the corresponding effect of their combination in doses $\mathrm{d}_{\mathrm{A}}$ and $\mathrm{d}_{\mathrm{B}}$ can be obtained if $\left(\mathrm{d}_{\mathrm{A}} / \mathrm{D}_{\mathrm{A}}\right)+\left(\mathrm{d}_{\mathrm{B}} / \mathrm{D}_{\mathrm{B}}\right)=1.0$. Thus if in a real experiment this sum is $>1.0$ or $<1.0$, it points to antagonism or synergism, respectively.

It is very popular to represent this paradigm with a graphic analog called Loewe isobole, the general form of which for different variants of combined toxicity was demonstrated, for example, by Yeh et al. [3] (Fig. 1b) together with a bar diagram corresponding to the Bliss independence paradigm (Fig. 1a). On the axes of the isoboles, the concentrations of both substances are plotted in relation to corresponding minimum effective values. Note that isoboles of the same type may be plotted on axes representing doses or concentrations in corresponding physical units (for example, $\mathrm{mg}$ or $\mathrm{mg} / \mathrm{l}$ ), the straight line describing additivity being drawn between points on the axes corresponding to isoeffective doses.

In the toxicological literature, the same two paradigms of combined effect are sometimes designated with the terms "heteroadditivity" (for Bliss independence) and "isoadditivity" (for Loewe additivity) or, accordingly, «effect additivity» and «dose additivity». We prefer the latter terminology [4] as it is purely phenomenological and does not involve the complicated question of the character and mechanisms of interaction, for answering which data are often insufficient. Moreover, for practically any substance to have an effect, particularly in chronic intoxication, an important role belongs to many, rather than one biological site of this effect and/or its various mechanisms, some of which may be the same with regard to the substances considered in combination (which should provide isoadditivity) and others not the same (heteroadditivity). It has also been repeatedly pointed out that the correspondence of actual data to this or that of the combined toxicity models depends essentially on the shape of the dose-effect (or doseresponse) curve for an isolated effect of each substance and what segment of this curve the added effect of the second substance is considered on (for example, [3-5]). We are therefore inclined to regard Bliss independence vs Loewe additivity as two methods for estimating combined toxicity rather than two essentially different types of the latter. The use of both models was recommended by the so-called Saariselkä Agreement [6].

However, all of this theoretical and terminological confusion is still creating considerable difficulties for dealing with practical challenges involving the assessment of health risks posed by a combined impact of two or more substances. Thus, the generally accepted methodology of risk assessment recommends summing risks up where they are those of the same outcome, proceeding, in fact, from effect additivity (Bliss independence). The possibility of risk super-additivity or sub-additivity is actually ignored, leaving it in the zone of numerous uncertainties of this assessment. Meantime, in the same country where the fundamentals of this methodology have been established by the US Environmental Protection Agency, another authoritative organization, the American Conference of Governmental Industrial Hygienists, has been for decades determining the concentration of a mixture of several substances of similar toxicological effect as meeting the threshold limit values (TLVs) established for them only if their concentrations (C) correspond to the equation $\mathrm{C}_{1} / \mathrm{TLV}_{1}+\mathrm{C}_{2} / \mathrm{TLV}_{2}+\mathrm{C}_{3} / \mathrm{TLV}_{3}+$ $\ldots . .=1$. A similar approach to «same-effect substances» is also adopted in the Russian standards for maximum permissible concentrations of harmful substances in the air. It is easy to see that this approach complies with the paradigm of dose additivity (Loewe additivity) if one assumes that TLVs are really isoefective.

Note also that the definitions of basic concepts of combined toxicity such as «additive», «more than additive (potentiation, synergy)» and «less than additive (antagonism)», which were developed back in 1981 by a special WHO Expert Committee, fully comply with the paradigm of effect additivity (i.e. Bliss independence) [7]

It should be added to the said above concerning the ambiguity of the definitions in this field of research that even within the framework of one of the two paradigms under consideration estimates of the combined toxicity of some two substances may essentially differ depending on the extent of impact and its duration, on which of the components of a combination prevails in it quantitatively, and, finally, on the organ or the system of the organism to which the effect considered pertains [4]. We will illustrate this statement with an example showing, with the help of Loewe isoboles, a two-phase character of combined toxicity depending on the ratio of two substances in a toxic combination (by their lethal effect) (Fig. 2).

Meanwhile, what is expected from a toxicologist is a clear and quite concrete recommendation concerning a rule for monitoring safe concentrations of chemical substances in the environment or for assessing health risks posed by the impact of these substances at unsafe levels in view of their combined effect. It is therefore that a proposal was put forward to establish an auxiliary concept of "main" or 

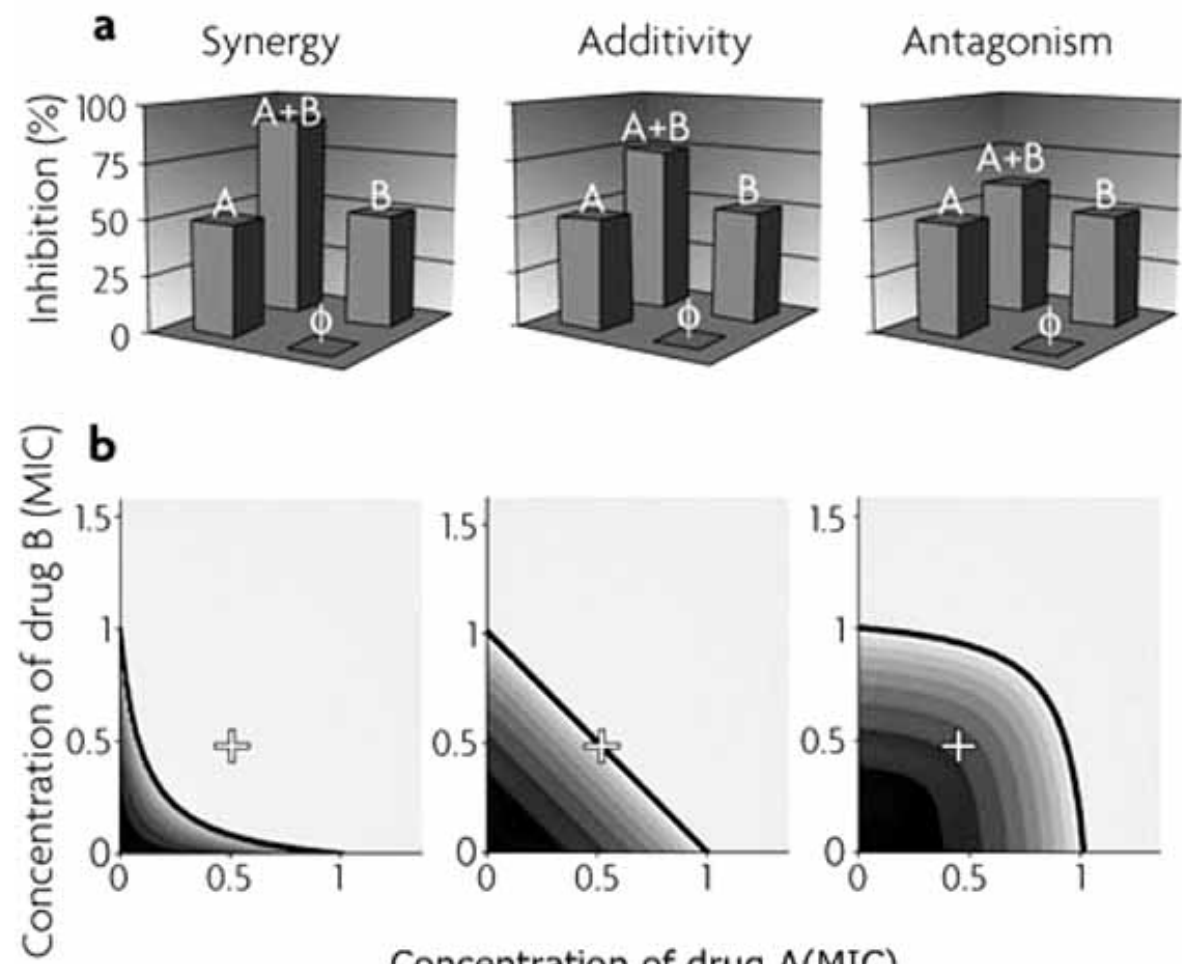

Concentration of drug $A(M I C)$

Fig. (1). Graphic presentation of Bliss independence (a) and Loewe additivity (b) for bacterial growth inhibition by 2 different drugs; MIC = minimum inhibitory concentration; $\varphi$ represents no drug. Adapted by permission from Macmillan Publishers Ltd: Nature Reviews Microbiology [3] (License Number 2346381014201, Jan 12, 2010).

"determinant" type of combined effect for resolving such issues $[4,8]$. It was recommended that the choice of the main type of combined toxicity should be based on:

- $\quad$ the predominant significance of the type of combined effect that is revealed for doses (concentrations) causing chronic intoxication, and for doses which are close to the lowest observed adverse effect level (LOAEL);

- $\quad$ in cases where the combination under consideration occurs in real conditions mainly in a narrow range of ratios between its components - the priority given to the type of combined effect that is characteristic of this range;

- $\quad$ in cases where the organs and systems the response of which is the most involved in toxicodynamics and toxicokinetics of combined intoxication are known the priority given to the type of combined effect that dominates with regard to changes in these organs (systems);

- in cases where the substances making up the combination, or at least one of them, are especially harmful to the organism or population (in particular, when carcinogenicity, mutagenicity, influence on the reproductive function are involved)-the priority given to the type of combined effect that is observed for these harmful effects, particularly in cases of synergy.

(However, outside the scope of hygienic standard setting and risk assessment it is not only the main type but also the

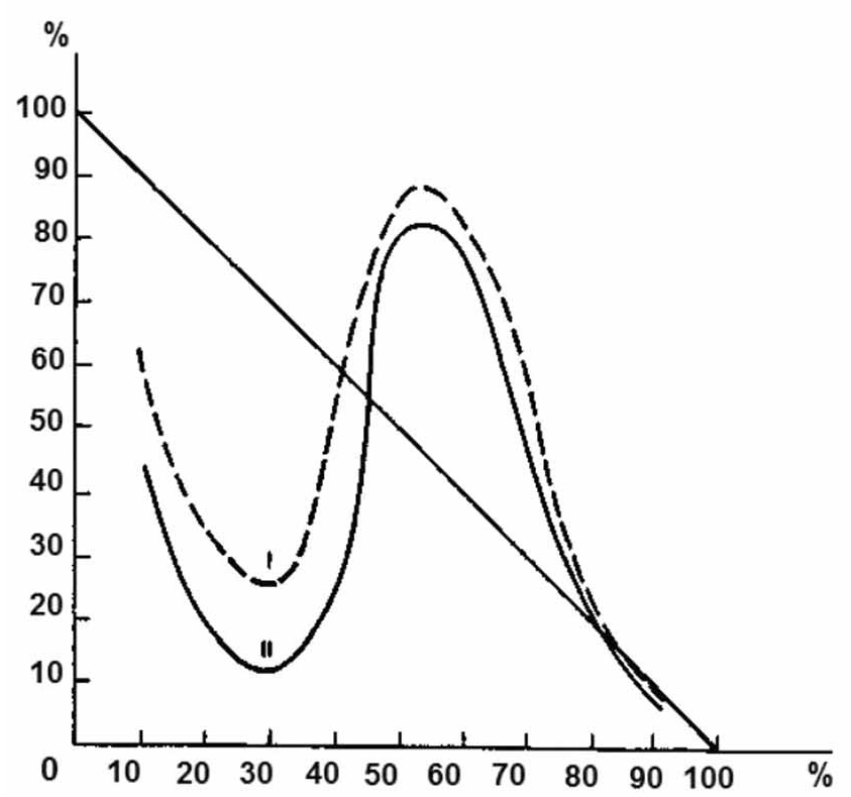

Fig. (2). Isoboles of combined acute lethal effect of sodium fluoride (the axis of abscissas) and manganese chloride (the axis of ordinates) for mice (curve I) and for rats (curve II). Concentrations are expressed as $\%$ of corresponding $\mathrm{LD}_{50}$. The straight line corresponds to dose additivity (from [4]-based on experiments carried out by late Vera I. Davydova).

entire range of possible types of combined toxicity are of both theoretical and practical interest. For instance, even if additivity is accepted as the main type of combined toxicity 
based on the above criteria, the possible attenuation of certain manifestations of intoxication as a result of toxicological antagonism inherent to them can blur the clinical picture and modify the doctor's attitude to the diagnostic value of corresponding symptoms and, possibly, therapeutic tactics).

Given the above described complexity of the problem and the admissible degree of uncertainty inherent in risk assessment generally, for the problem at issue to be solvable practically it should be considerably simplified not only by focusing it on the determinant (main) type of combined toxicity but also in some other important aspects.

First. As one of the basic stages of risk assessment (analysis of dose-response relationship) rests on both experimental toxicological and epidemiological data (the latter being preferable, in principle, but the former being used a lot more often for the majority of hazards in view of the shortage of epidemiological data), it is desirable to unify the concepts and terminology within the framework of theory and practice of risk assessment taking as a basis those accepted in the toxicological literature. At the same time, considering the priority of epidemiological human data, it is essential to find simple approaches to assessing combined effects on the basis of just these data.

Second. Given the fact that even a prospective (for example, cohort) epidemiological study is extremely difficult to organize as an experiment of strictly quantitative design, let alone the often inevitable need to use retrospective data, the problem of choice between effect additivity and dose additivity as the principal paradigm of combined risk analysis may hardly be resolvable on an a priori basis. This choice would rather be empirical, based on available material, and it is quite admissible to combine the two approaches.

Third. It is hardly realistic and even reasonable to try and estimate quantitatively the degree to which a combined risk deviates from a summative one. Even if we managed to establish, based on Loewe additivity, that under certain conditions of exposure the effective sum of doses expressed as ratios to respective isoeffective doses of two or more hazards is equal not to $1.0 \mathrm{but}$, for instance, to 1.2 , it would be rather imprudent to accept exactly the $20 \%$ reduction in the simple sum of risks when assessing them under similar conditions. We believe that uncertainty in risk assessment in such cases would be substantially reduced already if an expert had grounds to say, for example, that "Given toxicological antagonism, as shown not only in animal experiments but also on the basis of epidemiological analysis, as the main type of the combined action produced by given hazards, we can accept that simple summation of risks is a sufficiently conservative approach, overestimating rather than underestimating the combined risk». Or, alternatively, «Given toxicological synergy, as shown not only in animal experiments but also on the basis of epidemiological analysis, as the main type of the combined action produced by given hazards, we can accept that simple summation of risks is most likely to underestimate the combined risk». Although in so doing the expert would merely specify the sign of the uncertainty, we believe this alone would represent a substantial enhancement of the reliability of assessment as a whole.
Fourth. If one accepts the first three statements, it should be clear that very complex and sophisticated mathematics often involved in theoretical analysis of combined toxicity (for example, $[9,10]$ ) is hardly suitable for analyzing real data, epidemiological in particular, and for applying the outcomes of such analysis to practical risk assessment.

In this paper, we would like to offer a specific example of a solution to the problem based on the above considered principles using our own earlier published epidemiological data on the dependence of preclinical kidney damage in children on environmentally caused exposure to lead and cadmium estimated by the concentrations of these metals in urine [11]. Both metals are polytropic (poly-organ and polysystem) poisons but kidneys suffer from both lead and cadmium chronic intoxication. The low levels of exposure that are associated with environmental pollution in the vicinity of nonferrous metallurgy sites have been shown to pose a risk of child nephropathy by many researchers [11 16] and, moreover, is most likely to be one of the key risks. Therefore, according to the criteria suggested above, the type of environmentally caused combined nephrotoxicity of lead and cadmium falls within the concept of «main (determinant) type».

\section{MATERIAL AND METHODS}

A. The source of epi data used for our analysis was a study carried out by our team in 2004-2005 [11]. In that study, 3 to 7 years old preschool children from four towns in the Middle Urals, three of which are located close to copper refineries, were examined by qualified pediatricians-nephrologists. Only the children who had no history of explicit renal disease and were in generally good health were included into the study. Morning urine concentrations of cadmium $(\mathrm{Cd})$ and lead $(\mathrm{Pb})$, and of beta-2-microglobulin (B2u) were measured in 273 children. A case-control study design was used to evaluate the association between $\mathrm{B} 2 \mathrm{u}$ levels and body burdens of $\mathrm{Pb}$ and $\mathrm{Cd}$ as measured by their urine levels. B2u levels ranged from 10 to $1200 \mu \mathrm{g} / \mathrm{L}$. "Cases" were arbitrarily defined as those with $\mathrm{B} 2 \mathrm{u}$ levels above the median of the whole data distribution and "controls" as those at or below the median. The adjusted odds ratios per $\mu \mathrm{g} / \mathrm{L}$ of metals were: 1.07(1.01-1.13, $\mathrm{P}<0.02)$ for $\mathrm{Cd}$ and 1.02(1.01-1.03, $\mathrm{P}<0.0001)$ for $\mathrm{Pb}$. The results obtained in the study suggested that urine cadmium and lead concentrations characteristic of children living in polluted areas might be associated with incipient (mostly sub-clinical) renal damage in some of them. In that study only the urine beta 2microglobulin (B2u) level was chosen as a well established marker of tubular damage widely used by different researchers and sensitive for detecting renal damage induced by cadmium or lead. The results of investigations that compared different renal damage markers in children environmentally exposed to cadmium and lead were contradictory and gave no reliable criteria for a better choice. The authors did not adjust either $\mathrm{B} 2 \mathrm{u}$ or $\mathrm{Cd}$ and $\mathrm{Pb}$ levels for creatinine concentration because such adjustment, based on a common denominator for all three renal 
indices, would not change the quantitative relationships between them. (More about these study limitations with relevant references see in Discussion.)

B. Analysis based on the paradigm of effect additivity. When using the merged dataset obtained in the above study, and for the purpose of analyzing the combined effect of lead and cadmium, we excluded 13 children for whom information was incomplete, and thus we had a set of 260 children. The first step in analysis was the translation of metal concentrations into categorial form. To this end, following the ordering of entries in the dataset in the ascending order of cadmium (or lead) concentrations, all of the corresponding series was divided into three, roughly equal groups (86-87 children in each) corresponding to three levels of cadmium (or lead) action on kidneys conventionally designated as low, medium and high (assuming that the urine concentration of metal may serve as a bio-marker of this action). The mean concentrations were: (a) for lead, "low" level is 10.5, "medium " is 37.9, and "high" is $74.6 \mu \mathrm{g} / \mathrm{l}$; (b) for cadmium, 0.5, 2.3 and $9.8 \mu \mathrm{g} / 1$, respectively.

Then for each level of action of this or that metal we calculated the probability $\left(\mathrm{W}_{\mathrm{ij}}\right)$ of being referred to "cases" (i.e. the probability that the value of $\mathrm{B} 2 \mathrm{u}$ concentration exceeds the median for the entire dataset) for each of the 3 levels of action of the other metal. The results of these calculations were considered in both tabulated and graphic form, but in this paper, for the sake of simplicity and greater clarity, we present it in the latter form only.

In the notation used, indices $i=0,1,2$ and $j=0,1,2$ designate cadmium and lead groups, respectively. Thus, we introduce the following notation for probability $\mathrm{W}_{\mathrm{ij}}$ :

\begin{tabular}{|c|c|c|c|}
\hline \multicolumn{1}{|c|}{$\boldsymbol{P d}$} & $\mathbf{P b}=\mathbf{0}$ & $\mathbf{P b}=\mathbf{1}$ & $\mathbf{P b}=\mathbf{2}$ \\
\hline \hline $\mathrm{Cd}=0$ & $w_{00}$ & $w_{01}$ & $w_{02}$ \\
\hline $\mathrm{Cd}=1$ & $w_{10}$ & $w_{11}$ & $w_{12}$ \\
\hline $\mathrm{Cd}=2$ & $w_{20}$ & $w_{21}$ & $w_{22}$ \\
\hline
\end{tabular}

This table can be considered as an ANOVA two-way table for two three-level factors. Then the condition of a lack of factor interaction at all their levels leads to the following set of equations, which can be considered as a condition of effect additivity for the action of the factors at all levels:

$$
\left\{\begin{array}{l}
w_{22}-w_{21}=w_{12}-w_{11} \\
w_{22}-w_{20}=w_{12}-w_{10} \\
w_{22}-w_{21}=w_{02}-w_{01} \\
w_{22}-w_{20}=w_{02}-w_{00}
\end{array}\right.
$$

This set generalizes the equality $w_{11}+w_{00}=w_{10}+w_{01}$ which corresponds to the Bliss independence if we assume that probabilities $\mathrm{w}_{\mathrm{ij}}$ are low.
The effects of cadmium and lead are recognized as additive if all of the four equalities are fulfilled simultaneously, and a statistically significant deviation from even one of them means super- or sub-additivity of effects at this or that level of action. The statistical significance of the nonadditivity (for example, if the 4-th condition is not observed in set (1)) is verified with the help of the inequality:

$$
\left\{\frac{\left|\left(\frac{d_{22}}{n_{22}}-\frac{d_{20}}{n_{20}}\right)-\left(\frac{d_{02}}{n_{02}}-\frac{d_{00}}{n_{00}}\right)\right|}{\sqrt{\frac{d_{22}\left(n_{22}-d_{22}\right)}{n_{22}^{3}}+\frac{d_{20}\left(n_{20}-d_{20}\right)}{n_{20}^{3}}+\frac{d_{02}\left(n_{02}-d_{02}\right)}{n_{02}^{3}}+\frac{d_{00}\left(n_{00}-d_{00}\right)}{n_{00}^{3}}}}>Z_{\frac{1-\alpha}{2}}\right\}
$$

where $n_{i j}$ is the total number of children in group; $i j, d_{i j}$ is the number of children in the group with high $\mathrm{B} 2 \mathrm{u}, Z_{\underline{1-\alpha}}$ is the level $\frac{1-\alpha}{2}$ quantile of standard normal distribution $\mathrm{N}$ $(0,1)$.

\section{Analysis Based on the Paradigm of Dose Additivity}

We shall carry out this analysis by constructing a Loewe isobole. For constructing it as a line of equal values of $\mathrm{W}$ we use the multiple regression equation in quadratic form (3):

$\mathrm{W}=\mathrm{b}_{0}+\mathrm{b}_{1} \mathrm{Cd}+\mathrm{b}_{2} \mathrm{~Pb}+\mathrm{b}_{3} \mathrm{Cd} * \mathrm{~Pb}+\mathrm{b}_{4} \mathrm{Cd}^{2}+\mathrm{b}_{5} \mathrm{~Pb}^{2}$

where $\mathrm{Cd}$ and $\mathrm{Pb}$ are cadmium and lead concentrations, $\mathrm{b}_{0}$, $b_{1}, \ldots, b_{5}$ are regression coefficients. If we assume in expression (3) that $\mathrm{W}=$ const, then equation (3) presents an implicit equation of a certain curve on the $\mathrm{Cd}, \mathrm{Pb}$ axes, which is just the sought-for isobole. The points of intersection of this isobole with the axes correspond to the values of the isoeffective concentrations for cadmium or lead acting separately. Recall that in our case $\mathrm{W}=0.5$ is the most natural value of $\mathrm{W}$ because the boundary $\mathrm{B} 2 \mathrm{u}$ level dividing "cases" and "controls" was assumed to be the median of the distribution of these levels.

For constructing a regression equation expressing $\mathrm{W}$ as a function of cadmium and lead concentrations, it is necessary to have a set of values of $\mathrm{W}$ obtained for different $\mathrm{Cd}$ and $\mathrm{Pb}$ values. For this purpose, the primary dataset containing information on the lack or presence of an elevated (over the median value) concentration of microglobulin $B 2 u$ in each child is ordered by the magnitude of cadmium concentration, and all entries are divided into a certain number of parts equal to the number of entries, for example, into 9 parts (29 entries in each part). Let us remove from such ordered dataset the first 29 entries, and calculate $\mathrm{W}$ and mean values for cadmium $<\mathrm{Cd}>$ and lead $<\mathrm{Pb}>$ in the remaining dataset. These three quantities ( $\mathrm{W},<\mathrm{Cd}>,<\mathrm{Pb}>$ ) will be the first entry of the dataset required for constructing a regression. Next we return the 29 removed entries back in place and remove 29 entries located in the second part of the ordered full dataset, and for the sample thus created we again calculate $\mathrm{W}$ and the mean values for cadmium and lead. These three quantities will be the second entry of the dataset required for constructing a regression. This manipulation is repeated 9 times (according to the number of divisions of the 
primary dataset), and thus we obtain 9 entries for the dataset for constructing regression (3).

By carrying out similar operations for the primary dataset ordered with regard to lead content, we obtain 9 more entries. Finally, we add to the dataset for regression 4-5 entries obtained by eliminating from the primary dataset the numbers of entries that exceed 29. This is necessary for obtaining several values of $\mathrm{W}$ greatly differing from $\mathrm{W}=0.5$.

Using the dataset obtained, we select by the method of stepwise forward regression the predictors that are most important for describing W. Out of the five predictors of equation (3) the stepwise forward regression method selected three first ones (two linear terms and a cross term) for which $\mathrm{p}<0.05$; the multiple regression correlation coefficient proved to be equal to $\mathrm{R}^{2}=0.95$ (very high). The two quadratic terms of equation (3) proved to be statistically insignificant (p>> 0.05) and, therefore, were excluded. Thus, we obtained regression equation (4), presented below in section Results along with the isobole constructed on the basis of this equation (Fig. 5).

\section{RESULTS}

Figs. $(3,4)$ show graphically the dependencies of $\mathrm{W}_{\mathrm{ij}}$ on the lead content of the urine for various levels of cadmium concentrations and, vice versa, on cadmium concentrations for various levels of lead concentration.

Judging by the Spearman's rank correlation coefficients $\left(\mathrm{r}_{\mathrm{s}}\right)$ computed for the dependence of the effect on lead concentration ( 0.25 for low, 0.25 for medium and 0.21 for high cadmium concentrations) and corresponding $p$-values $(0.018,0.021$ and 0.049$)$ the general trend towards effect enhancement with growth in the impact of lead is statistically significant at any cadmium level. On the contrary, for the dependence of the effect on increasing cadmium levels the corresponding trend is significant only at low lead levels $\left(\mathrm{r}_{\mathrm{s}}=0.25, \mathrm{p}=0.022\right)$; at medium levels it becomes weaker $\left(\mathrm{r}_{\mathrm{s}}=0.13, \mathrm{p}=0.22\right)$, disappearing at high levels $\left(r_{s}=0.02, p=0.88\right)$.

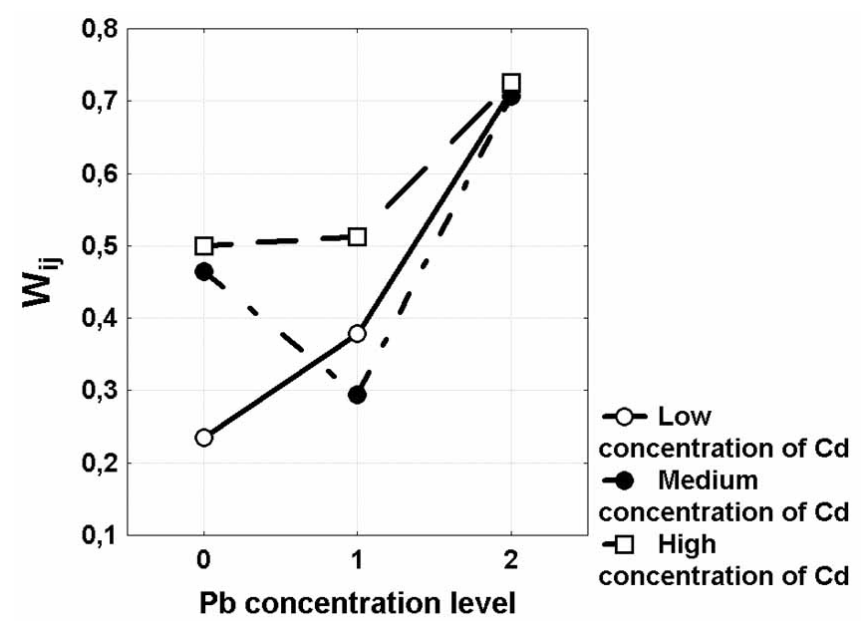

Fig. (3). Dependence of the probability $\left(\mathrm{W}_{\mathrm{ij}}\right)$ that $\mathrm{B} 2 \mathrm{u}$ level exceeds the median value on urine lead concentration level at various levels of cadmium concentration.
Regression equation (3) transformed in the course of the stepwise exclusion of variables as described in the previous Section has acquired the following form:

$\mathrm{W}=0.160+0.015 \mathrm{Cd}+0.008 \mathrm{~Pb}-0.0003 \mathrm{Cd} * \mathrm{~Pb}$.

Assuming $\mathrm{W}=0.5$, we obtain points that permitted us to construct the isobole shown in Fig. (5). The straight line drawn between the points where the curve crosses the axes of coordinates corresponds to dose additivity hypothesis.

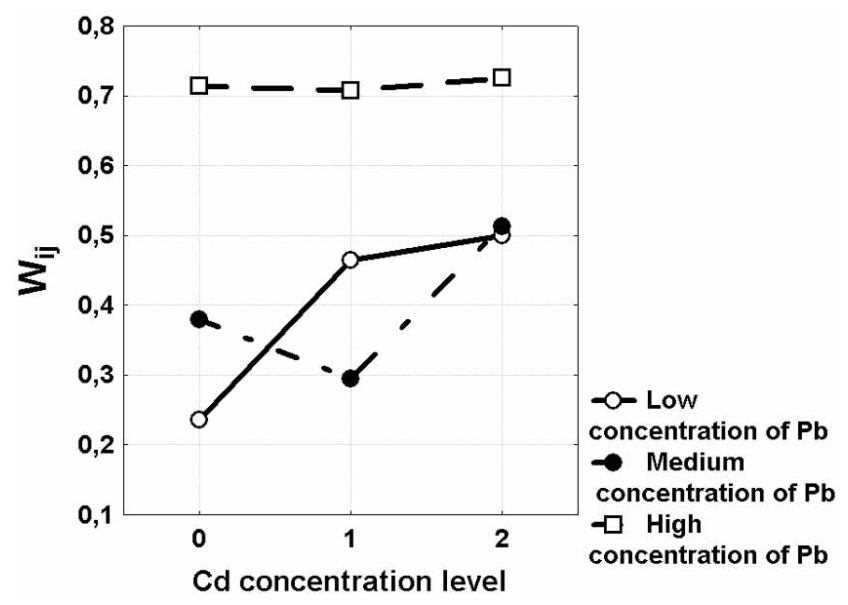

Fig. (4). Dependence of the probability $\left(\mathrm{W}_{\mathrm{ij}}\right)$ that $\mathrm{B} 2 \mathrm{u}$ level exceeds the median value on urine cadmium concentrations at various levels of lead concentration.

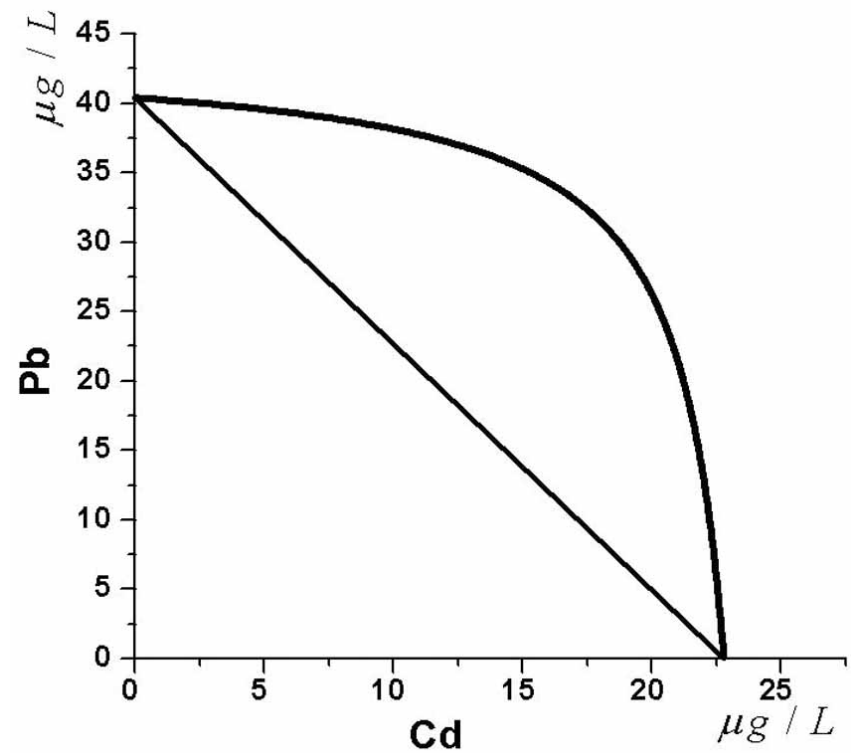

Fig. (5). An equal probability isobole for the above-median value of the microglobulin B2u concentration against lead and cadmium concentrations.

\section{DISCUSSION}

Although the nephrotoxicity of both cadmium and lead has been well established both experimentally and epidemiologically, most of the epidemiological studies assessed risks for highly exposed workers or for adults dwelling in polluted areas focusing on either cadmium [17- 
24] or lead [25, 26] as separate risk factors. The exceptions were the investigations [27] where a positive correlation between renal damage and blood lead levels was revealed in a cross-sectional epidemiological study designed to demonstrate the adverse effects of cadmium, and [28] dealing with the effects of concurrent exposure to cadmium and lead on renal function indices in nonsmokers.

Meanwhile, chronic nephro-urinary diseases in children cause a considerable public health concern in Russia due to their high prevalence, especially in some industrial regions such as the Middle Urals [11]. In this region, a lot of urban areas are extensively contaminated due to high emissions of lead, cadmium, and other pollutants. Cadmium and lead are emitted from copper smelters and other nonferrous metallurgical plants, and leaded gasoline was used until the early 2000 in this heavy traffic area. These two metals were considered as the most serious environmental nephrotoxins in the region. A risk assessment case study (in which doseresponse relationship was constructed by the authors based on published Japanese data on the association between elevated $\mathrm{B} 2 \mathrm{u}$ and cadmium levels in urine) indicated that evaluation of the nephrotoxic effects of cadmium in the area was warranted [29] but in that study the nephrotoxicity of lead was not taken into account. In general, there are but a few publications concerning renal damage in children due to environmental exposures to lead, and those dealing with such effects of cadmium are even scarcer.

Thus, Verberk et al. [13] demonstrated a positive relationship between the concentration of lead in blood $(\mathrm{PbB})$ and the activity of $N$-acetyl- $\beta$-D-glucosaminidase (NAG) in the urine of 151 children residing at different distances from a lead smelter in Romania. No relationship was found, however, between $\mathrm{PbB}$ and other renal-effect markers, such as urine albumin, alpha 1-microglobuline, retinol binding protein (RBP) or alanine aminopeptidase. On the contrary, Bernard et al. [14], who examined 51 children attending two schools in the vicinity of a lead smelter in Belgium and 51 from a school in a rural area, found but two significant inter-area differences, namely in $\mathrm{PbB}$ (but not in cadmium blood levels-CdB) and in the urine RBP (but not in NAG, albumin, beta 2-microglobuline and Clara cell protein). In a stepwise regression analysis, urine RBP excretion was found to be associated with $\mathrm{PbB}$ but not with $\mathrm{CdB}$.

De Burbure et al. [15] reported the results of testing blood and urine markers in 400 children and 600 adults living around two nonferrous smelters in northern France and in neighboring municipalities with unpolluted soil. Although both $\mathrm{PbB}$ and $\mathrm{CdB}$ levels were regarded by the authors as relatively low, $\mathrm{PbB}$ was significantly increased in boys, girls, and women living in the polluted area, while $\mathrm{CdB}$ was higher in boys, men and women living there. In the meantime, none of the studied renal-effect urine parameters (total protein, albumin, beta 2-microglobulin, NAG, RBP, brush border protein) showed a significant difference between the exposed and control groups, and the only positive correlation found in the whole of the children population was that between NAG in urine and CdB levels. Later on, however, when the population under consideration was extended to $>800$ children by including into the study some areas around historical nonferrous smelters not only in
France but also in the Czech Republic and Poland, it was found that urine excretion of NAG, RBP, and Clara cell protein was positively associated with $\mathrm{CdB}$ or urine $\mathrm{Cd}$ levels (and with urine $\mathrm{Hg}$ as well) [16] . As for the $\mathrm{PbB}$, it was negatively associated with serum levels of creatinine and of beta 2-microglobulin, suggesting, in the authors' opinion, renal hyperfiltration of these substances.

To the extent of our knowledge, the epidemiological study that provided materials for the present analysis was the first case-control study devoted to the problem of damage to kidneys in children due to the combined effect of cadmium and lead [11]. However, in order to evaluate renal damage resulting from relatively low urinary cadmium and lead levels, in that research children with explicit renal disease were excluded because such disease can arise from multiple causes that could confound the analysis and reduce the ability to detect renal damage due just to the impact of the metals. Thus the "cases" were not definitely ill children but those with comparatively higher values of a renal-effect marker. The underlying assumption was that higher values indicated a higher likelihood that a child had some degree of renal damage. Thus, in our present analysis we use an array of individual data in which each observation is described by three variables: concentration of lead in urine, concentration of cadmium in urine and preclinical quasi-diagnosis (i.e. pertaining or not pertaining to "cases" as defined in the Section "Material and methods").

The urine beta 2-microglobulin (B2u) level was used in that study as a well established marker of tubular damage widely employed by different authors and sensitive for detecting renal damage induced by cadmium $[18,24$ and many others) and lead [30]. The results of investigations that compared different renal damage markers in children environmentally exposed to cadmium and lead were contradictory [13-16] and gave no unequivocal criteria for a better choice.

Thus, again, cases were arbitrarily defined as children with B2u levels above the median for the whole dataset. Controls were defined as children with $\mathrm{B} 2 \mathrm{u}$ levels at or below the median value. Neither the metals nor B2u excretion levels were creatinine-adjusted in that study. This might limit the reliability of assignment of a child to cases or controls and reliance on the distribution of individual values. It could not, however, substantially influence the relationship between the values for the metals and $\mathrm{B} 2 \mathrm{u}$ because all three individual values (i.e. $\mathrm{Pb}, \mathrm{Cd}$, and $\mathrm{B} 2 \mathrm{u}$ concentrations) would be adjusted for one and the same creatinine concentration, while just this relationship is of interest for both former and present analysis of these data.

As it was stated in the "Material and methods" Section of this paper, the former study under consideration had demonstrated that both cadmium and lead urine concentrations characteristic of children living in polluted areas might be associated with incipient (mostly sub-clinical) renal damage in some of them. Later on, this conclusion was supported by positive results of anti-toxic intervention [31, 32]. There was, however, a question remaining unanswered, namely, how to estimate the character of the combined impact of these two metals on kidneys. The desire to answer this question - no doubt an important issue for assessing the risk of environmentally caused renal pathology in children- 
has encouraged us to undertake additional analysis of the same data. At the same time, this piece of research may be viewed as a development of the general methodology for estimating combined toxicity based on concepts accepted in experimental toxicology but rarely used in environmental epidemiology.

Estimation carried out with the help of inequality (2) has shown that the effect of a combination of lead and cadmium statistically significantly differs from a summative one. Important additional information for qualitative description of this difference at different levels of impact is provided by visual analysis of the curves in Figs. $(\mathbf{3}, \mathbf{4})$. It is easy to see that the dependence of the effect under consideration (i.e. the probability of an above median value of $B 2 u$ concentration) on the concentration of any of the two metals in urine is substantially different at different concentrations of the other metal. Thus, for example, the effect of lead clearly accrues from level to level only at low cadmium concentrations whereas at medium and high cadmium concentrations an increase in lead concentration enhances the effect only if we consider transition from medium to high lead level. The fact that growth in lead concentration at medium and high cadmium concentrations does not lead to effect enhancement (or even results in its reduction) suggests partial suppression of lead's nephrotoxic action by cadmium, i.e. antagonism.

Besides, as follows from Fig. (3), at low lead concentrations the increasing concomitant action of cadmium enhances the combined effect but does so to an approximately equal degree at medium and high levels of this action, i.e. the combined effect of the two metals is a little less than additive. At medium lead levels, only high cadmium levels enhance the combined effect whereas medium levels even reduce it a little. However, high concentrations of lead give an effect that is not dependent on the level of the concomitant action of cadmium, which does not contradict the possibility of a difference in the character of combined toxicity at different quantitative ratios between the components of the combination mentioned in the Introduction. Note that the nephrotoxicity of lead is clearly dominant at fairly high levels of both metals. This complies with our experimental data showing that at doses which are proportionately equal relative to corresponding $\mathrm{LD}_{50}$, chronic intoxication of rats with lead causes more severe damage to kidneys than cadmium [31]. In terms of combined toxicity, however, this "dominance" may mean the suppression of cadmium's contribution to the developing effect by lead, i.e. antagonism.

This manifestation of antagonism is even more pronounced in Fig. (4), showing the dependence of $\mathrm{W}_{\mathrm{ij}}$ on cadmium level at various lead concentrations. Thus, for example, we again can see that at high lead concentrations the combined effect is not only maximal but it is also independent of the additional contribution of cadmium, i.e. it is again subadditive (antagonism). Even for medium concentration of lead the combined effect is increased when the cadmium level goes from low to the high but not to the medium level. At low lead concentrations only, the antagonism to the action of cadmium does not fully suppress the gradual rise in the combined effect with growth in cadmium concentration.
It should be noted that we have tested this approach not only for the division of the dataset into three equal parts as described in "Material and Methods" but also for other variants of division, shifting the boundary between "medium" level 1 and adjacent levels 0 or 2 to either side. Although the resulting plots were somewhat different, the relationship between them remained essentially the same. Such consistency of the outcome may be regarded as an additional evidence of its reliability.

The shape of the isobole shown in Fig. (5) confirms reciprocal antagonism in the nephrotoxic action of lead and cadmium (compare with Fig. 1b). Cadmium and lead concentrations corresponding to the points of intersection between the isobole and the axes of coordinates may be regarded as isoeffective for the separate action of a corresponding metal; for lead it is about $40 \mu \mathrm{g} / \mathrm{L}$, and for cadmium about $23 \mu \mathrm{g} / \mathrm{L}$. It should be borne in mind, however, that under equal exposure conditions cadmium is noticeably more toxic than lead: for example, at a single intraperitoneal injection of dissolved salts of these metals to rats $\mathrm{LD}_{50}$ of cadmium was found to be equal to $70 \mathrm{mg} / \mathrm{kg}$, and $\mathrm{LD}_{50}$ of lead to $220 \mathrm{mg} / \mathrm{kg}$ of body weight [31]. Hence we can assume that the effective lead concentration $\left(\mathrm{EC}_{50}\right)$ that we have found now with the help of an isobole was, in its «toxicological equivalent», not higher but somewhat lower than $\mathrm{EC}_{50}$ for cadmium. As mentioned above, it was shown in an experimental study as well that, being in equal proportions of $\mathrm{LD}_{50}$, lead produces a much stronger damaging effect on kidneys than cadmium [31].

The fact that the two independent approaches to analysis that we used have yielded mutually corroborating outcomes, which may be called model insensitivity of the main result, strengthens the validity of the conclusion of the non-additive character of lead and cadmium action on kidneys, the principal significance (at least, in the available range of exposures) pertaining to toxicological antagonism between lead and cadmium.

It should be stipulated, however, that although in the description of the analysis technique and results we dared to designate the two approaches employed as «analysis based on effect additivity paradigm» and "analysis based on dose additivity paradigm», we are well aware that we should be cautious about drawing an analogy to the corresponding concepts of experimental toxicology. An epidemiological study, even of "case-control" design, will be far from the possibilities of strict planning provided, for example, by $2^{2}$ or, at least, $2^{1}$ experimental design; moreover, the very techniques of our analysis are conspicuously unusual, in particular, the Loewe isobole method.

Nevertheless, we can maintain that the fundamental correspondence of our methods to the above paradigms cannot be denied. Our results may therefore be also regarded as an argument in favor of the idea formulated in the Introduction that the so-called «Bliss independence» and «Loewe additivity» present different models rather than really different mechanisms of combined toxicity, a fairly complex process involving toxicodynamic and toxicokinetic interactions at various levels of the organism [4]. It was also noted in the Introduction that this or that model may prove to be more suitable in specific conditions, but there may be a dataset for which both of them would be quite adequate, and 
the independence of the judgment about the main type of combined toxicity from the choice of the model can serve as evidence in favor of reliability of this judgment. We will not be able to claim that such independence is generally characteristic of epidemiological data analysis until sufficient experience has been gained in the use of similar approaches for analyzing data of other epidemiological studies of different designs, but it is quite possible to recommend the trial of both models in each such study.

It is quite clear that these particular approaches are directly applicable only to studies where the health outcome, i.e. the dependent variable is not continuous (quantitative) but is categorial (in particular, binary), and where the response may be given in terms of a probability of presence or absence of this or that category. However, the translation of any variable which is continuous by nature into a categorial one (as we have done in relation to $\mathrm{B} 2 \mathrm{u}$ concentration) is not only convenient and admissible for the purpose of data analysis but is also fully consistent with diagnostic principles (suffice it to mention the diagnostically relevant designation of body temperature as "normal" or "elevated".) As for the translation of explanatory variables (in our case, lead and cadmium concentrations in the urine of children) into categorial form, for numerically limited datasets it is an adequate method for elimination (or, at least, substantial limitation) of noise associated with their random scattering and facilitates more accurate identification of association between response and such variables.

\section{CONCLUSIONS}

Higher reliability of epidemiological data with regard to hazard identification and analysis of dose-response relationships compared with data obtained in animal experiments is one of the postulates of the general health risk assessment methodology. In the case of two or more hazards causing the same adverse effect, this principle ought to be extended to the concluding stage of such assessment, i.e. risk characterization if an expert assesses the type of combined risk. However, since the use of animal experimental data is still inevitably prevalent at all the above mentioned stages of risk assessment, it is reasonable to use for estimating combined toxicity based on the results of environmental epidemiological studies the concepts and terms that are well established in experimental toxicology, despite their certain intricacy and occasional inconsistency.

From this point of view, the best suitable concepts for epidemiological analysis of combined risks seem to be "additivity", "synergy" and "antagonism", and our work has shown (at least, in a «case-control» study) that sufficiently simple but adequate techniques make it possible to distinguish between these types of combined toxicity. It has been shown, in particular, that environmentally caused damaging action of lead and cadmium on kidneys in children is less than additive (a manifestation of toxicological antagonism).

Of theoretical interest is the demonstrated consistency of such assessment of the type of combined toxicity irrespective of whether it is carried out based on the effect additivity paradigm or the dose additivity one. We believe this allows us to speak in favor of regarding the so-called Bliss independence and Loewe additivity as complementary simplified models of the same fairly complex process rather than as essentially different phenomena. The example considered in the paper allows us to recommend testing approaches to epidemiological data analysis for the purpose of combined risk assessment using both models rather than restricting it to an a priori chosen one.

We believe that the specific practical value of the obtained results lies in the fact that, given the subadditivity (antagonism) of the damaging effect of lead and cadmium on kidneys, the summation of corresponding risks is a sufficiently conservative principle creating an additional margin of safety and limiting the uncertainty of risk assessment on the whole.

\section{REFERENCES}

[1] VanderWeele TJ. On the distinction between interaction and effect modification. Epidemiology 2009; 20: 863-71.

[2] Goldoni M, Johansson C. A mathematical approach to study combined effects of toxicants in vitro: evaluation of the Bliss independence criterion and the Loewe additivity model. Toxicol in vitro 2007; 21 : 759-69.

[3] Yeh PJ, Hegreness MJ, Aiden AP, Kishony R. Drug interactions and evolution of antibiotic resistance. Nat Rev Microbiol 2009; 7: 460-6.

[4] Katsnelson BA. The combined action of chemicals. In: Kurlyandsky BA, Filov VA, Ed. General Toxicology (Russian). Moscow: Meditsina Publishers 2002; pp. 497-520.

[5] Sühnel J. Zero interaction response surfaces, interactions functions and difference response surfaces for combinations of biologically active agents. Arzneimittelforschung 1992; 42: 1251-8.

[6] Greco W, Unkelbach HD, Pöch G, Sühnel J, Kundi M, Bödeker W. Consensus on concepts and terminology for combined-action assessment: The Saariselkä Agreement. Arch Complex Environ Stud 1992; 4: 65-9.

[7] WHO. Health Effects of Combined Exposures in the Work Environment. Geneva: World Health Organisation 1981.

[8] Katsnelson BA, Novikov SM. Methodological approaches to studying combined action of industrial noxious chemicals. Hygiene Sanitary (Russian) 1986; 8: 59-63.

[9] VanderWeele TJ, Robins JM. The identification of synergism in the sufficient-component-cause framework. Epidemiology 2007; 18: 329-39.

[10] VanderWeele TJ. Sufficient cause interactions and statistical interactions. Epidemiology 2009; 20: 6-13.

[11] Katsnelson BA, Kireyeva EP, Kuzmin SV, et al. An association between incipient renal damage and urine cadmium and lead levels in young Russian children: a case-control study. Europ Epi-marker 2007; 11: 1-8.

[12] Price RG, Patel S, Chivers I, Milligan P, Taylor SA. Early markers of nephrotoxicity: detection of children at risk from environmental pollution. Ren Fail 1999; 21: 303-8.

[13] Verberk MM, Willems TE, Verplanke AJ, De Wolff FA. Environmental lead and renal effects in children. Arch Environ Health 1996; 51: 83-7.

[14] Bernard AM, Vyskocil A, Roels H, Kriz J, Kodl M, Lauwerys R. Renal effects in children living in the vicinity of a lead smelter. Environ Res 1995; 68: 91-5.

[15] de Burbure C, Buchet JP, Bernard A, et al. Biomarkers of renal effects in children and adults with low environmental exposure to heavy metals. J Toxicol Environ Health 2003; 66: 783-98.

[16] de Burbure C, Buchet P, Leroyer A, et al. Renal and neurological effects of cadmium, lead, mercury, and arsenic in children: evidence of early effects and multiple interactions at environmental exposure levels. Environ Health Perspect 2006; 114: 584-90.

[17] WHO. ToxProfiles. Geneva: World Health Organization 2008; Available at: http://www.atsdr.cdc.gov/toxpro2.html

[18] Jarup L, Elinder CG, Spang G. Cumulative blood-cadmium and tubular proteinuria: a dose-response relationship. Environ Health 1988; 60: 223-9.

[19] Jarup L, Alfven N, Carlsson D, Elinder CG. Cadmium and tubular proteinuria. Epidemiology 1998; 9: 112. 
[20] Nogawa K, Kobayashi E, Honda R. Renal dysfunction in inhabitants in a cadmium-polluted area. Environ Res 1980; 23:1323.

[21] Nogawa K, Kido T, Yamada M. Alpha-1-microglobulin in urine as an indicator of renal tubular damage caused by environmental cadmium. Toxicol Lett 1984; 22: 60-3.

[22] Nogawa K, Kido T, Honda T. A dose-response analysis of cadmium in the general environment with special reference to total cadmium intake limit. Environ Res 1989; 48: 7-11.

[23] Nakagawa H, Kawano S, Okumura T. Mortality study of inhabitants in a cadmium polluted area. Bull Environ Contam Toxicol 1987; 38: 553-60.

[24] WHO. International Programme on Chemical Safety. Environmental Health Criteria 119. Principles and methods for the assessment of Nephrotoxity Associated with Exposure to Chemicals. Geneva: World Health Organisation 1991; p. 266.

[25] Campbell BC, Beattie AD, Moore MR, Goldberg A, Reid AG. Renal insufficiency associated with excessive lead exposure. BMJ 1977; 1: 482-5.

[26] Pocock SJ, Shaper AG, Ashby D. Blood lead concentration, blood pressure, and renal function. BMJ 1984; 289: 872-4.
Staessen J, Lauwerys RA, Bushet J-P. Impairment of renal function with increasing blood lead concentrations in general population. $\mathrm{N}$ Engl J Med1992; 327: 151-6.

[28] Satarug S, Nishijo M, Ujjin P. Evidence for concurrent effects of exposure to environmental cadmium and lead on hepatic CYP2A6 phenotype and renal function biomarkers in nonsmokers. Environ Health Perspect 2004; 112: 1512-8.

[29] Privalova LI, Wilcock KE, Keane SE. Some considerations concerning multimedia - multipollutant risk assessment methodology: use of epidemiologic data for non-cancer risk assessment in Russia. Environ Health Perspect 2001; 109: 1-7.

[30] Staessen J, Yeoman WB, Fletcher AE, et al. Blood lead concentration, renal function, and blood pressure in London civi servants. Br J Ind Med 1990; 47: 442-7.

[31] Kireyeva EP, Katsnelson BA, Degtyareva TD, et al. Nephrotoxicity of lead and cadmium and its inhibition by a complex of bioprotectors. Toxicological Review (Russian) 2006; 4: 26-32.

[32] Privalova L, Katsnelson B, Soloboyeva J, Kuzmin S, Kireyeva E, Degtyareva T. Additional evidence of association between exposure to lead and cadmium and renal disease in children. Epidemiology 2008; 18(Suppl 5): 12S

() Katsnelson et al.; Licensee Bentham Open.

This is an open access article licensed under the terms of the Creative Commons Attribution Non-Commercial License (http://creativecommons.org/licenses/by$\mathrm{nc} / 3.0 /$ ) which permits unrestricted, non-commercial use, distribution and reproduction in any medium, provided the work is properly cited. 